\title{
Obstructive Uropathy
}

National Cancer Institute

\section{Source}

National Cancer Institute. Obstructive Uropathy. NCI Thesaurus. Code C3675.

Uropathy that is caused by an impediment to flow in the urinary tract. 